\title{
Radiofrequency Ablation in Dense Ventricular Scar - Longer Continuous Lesions may be Beneficial
}

\author{
Jackson Liang ${ }^{1}$, Pasquale Santangeli ${ }^{2}$, and Francis Marchlinski ${ }^{3}$ \\ ${ }^{1}$ University of Michigan \\ ${ }^{2}$ University of Pennsylvania \\ ${ }^{3}$ Hospital of the University of Pennsylvania
}

April 27, 2020

\begin{abstract}
N/A (letter to editor)
\end{abstract}

We read with great interest the recent study by Rogers, et al. describing lesion formation with continuous versus intermittent radiofrequency ablation. ${ }^{1}$ The authors applied 50 (ex-vivo) or 10 (in-vivo) watts with either intermittent (15-seconds $\mathrm{x} 4,30$-seconds $\mathrm{x} 2$, or 60 -seconds $\mathrm{x} 1$ ) or continuous $(1,2,3$, or 5 minutes) radiofrequency applications and examined lesion size with each strategy. Continuous lesions resulted in significantly larger lesion size with possibly increased risk of steam pops at high power. In the ex-vivo model, they saw rapid lesion formation in the first minute of ablation with substantial drop-off in expansion of lesion size over time especially after 3 minutes (down to $0.35 \mathrm{~mm} / \mathrm{min}$ at 5 minutes), and suggest that there is only minimal incremental benefit of prolonging ablation lesion duration beyond 3 minutes in normal tissue.

They have, however, appropriately recognized the limitation of their model which lacks significant fibrosis, limiting the generalizability of their findings to ablation in patients with scar. It is important to recognize that lesion formation in scar-related ventricular tachycardia (VT) is likely to differ dramatically compared to normal myocardial tissue. Barkargan, et al. have shown in an in-vivo porcine model of anterior myocardial infarction that lesion formation in normal versus scar tissue can be substantially different. ${ }^{2}$ Specifically, ablation lesions in scar tissue were histologically quite heterogeneous, and connective tissue tended to be more resistant to thermal injury than normal myocardium.

We have seen many cases where after identifying critical VT circuit components with activation and entrainment mapping, VT slowing and termination could only be achieved very late (4-5 minutes) into radiofrequency application, suggesting continued lesion expansion due to delayed effect of conductive heating in scar tissue can persist well beyond 3 minutes. Historical data from Nath, et al. have demonstrated that tissue temperature of $>50^{\circ} \mathrm{C}$ must be reached in order to achieve nonreversible cellular damage. ${ }^{3}$ In the setting of dense scar, as in patients with healed myocardial infarction or dense basal septal scar in nonischemic cardiomyopathy, dynamics of tissue heating and lesion formation are likely quite different than in normal tissue. Especially in intramural substrates which are "protected" by dense subendocardial (or subepicardial if ablating from the epicardium) scar, ablation with very long ( $>3$ minute) lesions may have a more pronounced effect than in normal tissue. While in our experience, steam pops seem to occur less frequently when ablating in dense scar- even with long, high power lesions, close monitoring of ablation parameters including catheter tip temperature and impedance remain necessary during ablation to assure continued safety to avoid char formation and steam pops. 
We enthusiastically applaud the authors for their valuable contribution to the literature. While chronic cardiomyopathy models are technically difficult to create and costly to maintain, additional studies examining lesion formation in dense scar would be extremely helpful to delineate optimal ablation strategies in patient with cardiomyopathy and difficult VT substrates. Our clinical experience with late VT termination and prevention of inducibility after 4-5 minute radiofrequency ablation lesions in areas of marked fibrosis would support such additional study.

\section{References:}

1. Rogers AJ, Borne RT, Ho G, Sauer WH, Wang PJ, Narayan SM, Zheng L, Nguyen DT. Continuous Ablation Improves Lesion Maturation Compared with Intermittent Ablation Strategies. J Cardiovasc Electrophysiol 2020.

2. Barkagan M, Leshem E, Shapira-Daniels A, Sroubek J, Buxton AE, Saffitz JE, Anter E. Histopathological Characterization of Radiofrequency Ablation in Ventricular Scar Tissue. JACC Clin Electrophysiol 2019;5:920-931.

3. Nath S, DiMarco JP, Gallop RG, McRury ID, Haines DE. Effects of dispersive electrode position and surface area on electrical parameters and temperature during radiofrequency catheter ablation. Am J Cardiol 1996;77:765-767. 